\title{
Performance Improvement of Coded OFDM Communication System in AWGN Channel
}

\author{
Marwa Abdelfatah Abdeltwab \\ Instructor, Dept. of EECT, \\ Modern Academy in Ma'adi, \\ Egypt
}

\author{
K.A. ElBarbary \\ Professor and Head of Electric \\ Engineering Department, Suez \\ Canal University, Egypt
}

\author{
Adel Elhenawy \\ Professor, Dept. of Electrical \\ Communication, Ain Shams \\ University, Egypt
}

\begin{abstract}
OFDM (orthogonal Frequency Division Multiplexing) is a basic element in the $4 \mathrm{G}$ mobile system. The OFDM performance can be improved using the channel coding. The channel coding is a class of signal transformations designed to improve communication system performance.

The work presented in this paper discusses the performance improvement of OFDM communication system using different channel coding techniques through AWGN channel model. These coding techniques include Reed Solomon coding, Convolutional coding, Concatenated coding (by combining Reed Solomon with Convolutional), and Interleaved concatenated coding techniques. Besides, a new algorithm produced to choose a good convolutional encoder design for a certain rate and memory registers.
\end{abstract}

\section{Keywords}

OFDM, AWGN, Channel Coding, Reed Solomon, Convolutional, Interleaver.

\section{INTRODUCTION}

In recent years Orthogonal Frequency Division Multiplexing (OFDM) has gained a lot of interest in digital communication application. This has been due to its important features like robustness to multipath fading channel and high spectral efficiency [1, 2, 3]. OFDM is used in many digital applications as digital audio broadcasting (DAB), digital video broadcasting (DVB), Wireless Local Area Networks (WLAN), and other high speed data application for both wireless and wired communications [1,3].

OFDM overcomes most of the problems with older multichannel systems that use FDMA and TDMA. OFDM splits the available bandwidth into many narrow band channels. The carriers for each channel are made orthogonal to each other, allowing them to be spaced very close together, with no overhead in guard spacing between bands as in the FDMA technique, and there is no great need for users to be time multiplex as in TDMA, so that there is no overhead associated with switching between users. As each band is narrow, the symbol rate is low that limits the problem of multipath delay spread [4].

The channel coding is used to improve the performance of uncoded OFDM systems. There are many types of coding technique that used in OFDM as block code and convolutional code [5]. The concatenated code is a coding technique that uses two or more different codes to provide a large coding gain with less implementation complexity [6]. The interleaved concatenated code is the concatenated code with the use of an interleaver between the two coding techniques to achieve better improvement in the system performance [7].

The rest of the paper organized as follow:
Section 2 shows the concept of OFDM system. Section 3 shows the concept of different types of channel coding techniques that can be used in OFDM, including Reed Solomon, Convolutional, Concatenated, and interleaved concatenated codes. Section 4 shows the BER performance of uncoded and coded QPSK-OFDM over AWGN channel.

The simulation results presented in this paper demonstrate the advantage of systems with error control coding over uncoded system performances in terms of the coding gain.Finally Section 5 concludes the paper.

\section{OFDM CONCEPT}

Orthogonal Frequency Division Multiplexing (OFDM) is a communication technique that divides the available bandwidth into a number of equally spaced frequency bands. Each band has a subcarrier that carries a portion of the user information. Each subcarrier is orthogonal to each other, allowing overlapping between the bands without interference between them, and also cause saving of bandwidth as referred in Fig 1 $[1,8,9]$.

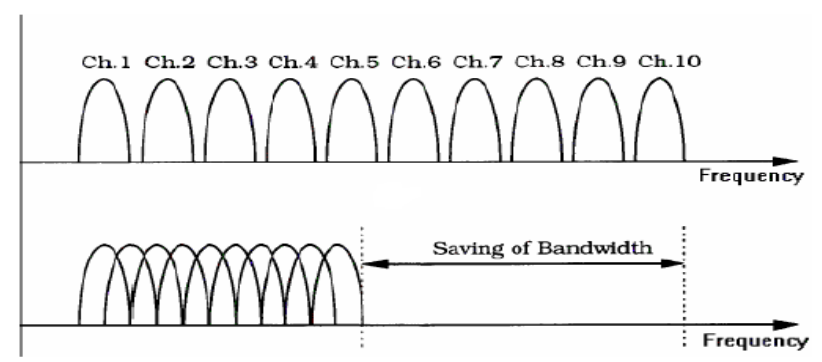

Fig 1: Saving Bandwidth using OFDM

Each carrier in an OFDM signal has a very narrow bandwidth; so that the resulting symbol rate is low (symbol time is large). This will give the signal a high tolerance to Multipath delay spread, because the delay spread must be very long to cause significant inter-symbol interference, and in order to totally eliminate ISI, OFDM employs a cyclic prefix which increases the length of the symbol period so that it is much greater than the delay spread of the channel $[3,4]$.

Fig 2 below shows the block diagram of an OFDM system [10].

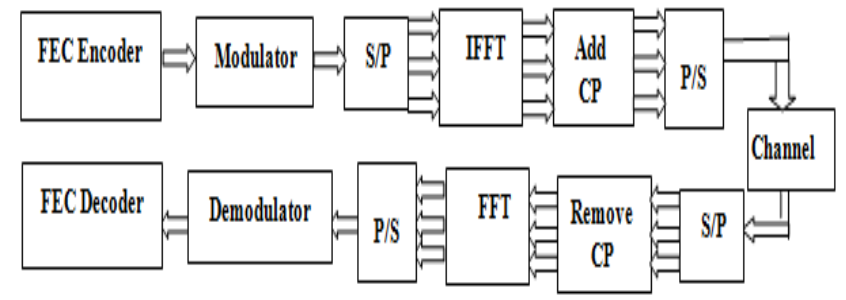

Fig 2: OFDM General Block Diagram 
The input data is first passed to the forward error correction (FEC) encoder to improve the system performance by adding redundant bits to the data. The coded signal is then sent to the modulator where the bits are mapped to symbols, which is represented in in-phase and quadrature (IQ) format.

The IFFT is then used for modulating the constellation mapped data onto the orthogonal subcarriers. The IFFT generally takes $\mathrm{N}$ symbols at a time where $\mathrm{N}$ represents the number of subcarriers in the system. Since the basis functions for an IFFT are $\mathrm{N}$ orthogonal sinusoids, all subcarriers will be orthogonal $[10]$. S/P and P/S converters used to adjust the format of the data depending on the input of the next block.

Finally a cyclic extension is added, and transmits through the channel.

\section{CHANNEL CODING}

Channel coding is the transformation of the signal in a certain designed method to enable the transmitted signals to better withstand the effects of various channel impairments such as noise, interference and fading [5].

\subsection{Reed Solomon Code}

Reed Solomon (RS) codes are nonbinary cyclic codes with symbols made up of m-bits sequences. The most conventional RS code with $(\mathrm{n}, \mathrm{k})=\left(2^{\mathrm{m}}-1,2^{\mathrm{m}}-1-2 \mathrm{t}\right)$ with $\mathrm{d}_{\min }=\mathrm{n}-\mathrm{k}+1$ [5].

Where $\mathrm{k}$ : number of data symbols, $\mathrm{n}$ : number of code symbols, m: number of bits in each symbol.

The RS encoder take $\mathrm{k}$ data symbols and add $2 \mathrm{t}$ redundant symbols to produce $\mathrm{n}$ code symbols where each symbols contain $\mathrm{m}$ bits [11]. The RS decoder has $2 \mathrm{t}$ redundant symbols which are twice the amount of correctable errors $t$, as for each error, one redundant symbol is used to locate the error and another redundant symbol is used to find its correct value [5].

The RS encoding process for the message word $\mathrm{m}(\mathrm{x})$, involves the following steps:

1. Generate the generating polynomial:

$$
G(x)=g_{0}+g_{1} x+g_{2} x^{2}+\ldots \ldots+g_{2 t-1} x^{2 t-1}+x^{2 t}
$$

2. Generate the parity polynomial:

$$
\mathrm{P}(\mathrm{x})=\mathrm{x}^{\mathrm{n}-\mathrm{k}} \mathrm{m}(\mathrm{x}) \text { modulo } \mathrm{G}(\mathrm{x})
$$

3. The required codeword polynomial will be:

$$
\mathrm{U}(\mathrm{x})=\mathrm{P}(\mathrm{x})+\mathrm{x}^{\mathrm{n}-\mathrm{k}} \mathrm{m}(\mathrm{x})[5]
$$

RS codes are designed to deal with the burst errors. As RS is a nonbinary code, it works with symbol based arithmetic rather than bit based arithmetic so that it can be configured with long block length (in bits) and large $d_{\min }$ with less decoding time, but this increase the complexity [5].

Shortening is performed to improve the performance of the RS code. It done by fixing the rate $(\mathrm{k} / \mathrm{n})$ and reduce both the message length (k) and the codeword length (n), so it have the same redundancy and the same error correcting capability as the original code but with less complexity, less decoding time and sometimes less power consumption. It is implemented by appending a certain number of zeros to the information message at the input of the original encoder then discarded these added zeros after the coding procedure [12].

\subsection{Convolutional Code}

A general convolutional encoder consists of $\mathrm{k}$ shift registers and $n$ modulo- 2 adders as shown in Fig 3 [5].

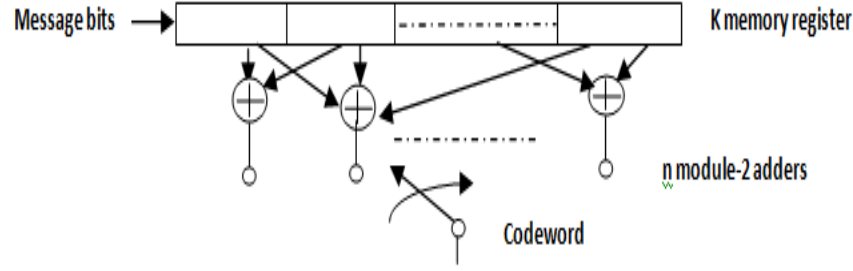

Fig 3: Convolutional Encoder

The message bits are shifted into the encoder one bit at a time, and the outputs of the $\mathrm{n}$ adders are sequentially sampled and transmitted, so that there are $\mathrm{n}$ code bits for each message bit and the code rate is $1 / \mathrm{n}$ [5]. So, the output sequence is function of both the current input bit and the k-1 previous bits [13]. The convolutional decoder uses the viterbi algorithm that is optimal as it performs maximum likelihood decoding. It reduces the computational load by taking the advantage of the special structure of the code trellis. As the trellis diagram represents the rules of the game as it describes all possible transitions and their corresponding state and finish states, so the error path must follow one of the allowable transitions. So, there are constraints in the encoding process, which cause the decoder to more easily meet good error performance using less $\mathrm{E}_{\mathrm{b}} / \mathrm{N}_{\mathrm{o}}$ [5].

The choice of connection between the adders and the registers in convolutional encoder provides a certain characteristics to the code. These connections are not chosen arbitrary, it must be chosen to yield good distance properties of the code, and it found by computer search for all possible connection to find the best. [5]

.The puncturing technique is used to improve the performance of a convolutional code, it obtained by puncturing or eliminating some outputs of the convolutional encoder. This technique increases the rate of the code and reduces the decoding complexity and time, but it reduces the distance of the code so reduce the error correcting capability of the code. [14]

\subsection{Concatenated Code}

A concatenated code is a very useful technique that leads to construct a powerful code that can provide large coding gain with less implementation complexity [10]. A big, powerful code with high BER performance can be constructed in an equivalent concatenated form by combining two or more codes of relatively small size and complexity to provide the same performance with less complexity, and decoding is done by combining two or more relatively low complexity decoders instead of a complex decoding of a big code [7].

\subsection{Serial Concatenated Code}

In a serial concatenated code, a message block is first encoded by the first code, normally called the outer code, then the output from the first code encoded by the second code, usually called the inner code. The decoding process operates in two stages: first performing the decoding of the inner code and then the decoding of the outer code. The decoding complexity decomposed into these two decoders is much lower than that of the direct decoding of the whole code as shown in Fig 4 [7, 15].

The concatenation between two codes will yield a new code with the following parameters:

The length $=\mathrm{n}_{1} * \mathrm{n}_{2}$

The minimum distance $<=\mathrm{d}_{\min 1} * \mathrm{~d}_{\min 2}$ 


\section{The rate $=\mathrm{R}_{1} * \mathrm{R}_{2}[7,15]$}

Where: the inner code has length $n_{1}$, minimum distance $d_{\min 1}$, and rate $\mathrm{R}_{1}$, and the outer code has length $\mathrm{n}_{2}$, minimum distance $d_{\min 2}$, and rate $R_{2}$.

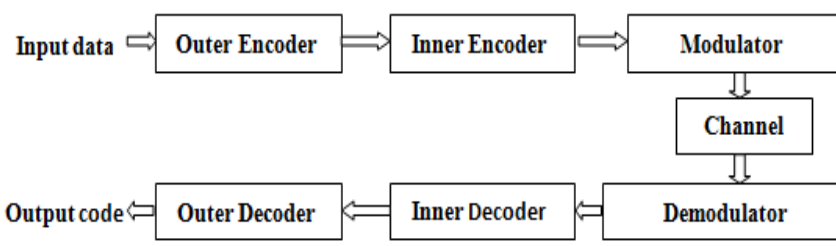

Fig 4: Serial Concatenated Code

\subsection{Interleaved Concatenated Code}

Interleaving is a technique that takes a given sequence of symbols and permutes their positions and arranging them in a different temporal order. Interleavers are used against burst errors, so it is designed to convert error patterns that contain long sequences of serial erroneous data into a more random error pattern, so distribute errors among many code vectors, Burst errors are characteristic of some channels, like the wireless channel, and they also occur in concatenated codes, where an inner decoder overloaded with errors can pass a burst of errors to the outer decoder [7]. Fig 5 represents interleaved concatenated code.

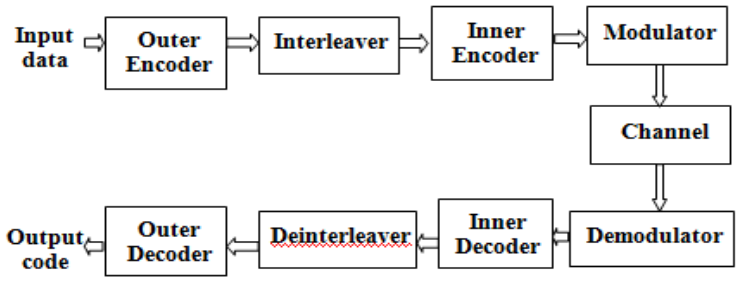

Fig 5: Interleaved Concatenated Code

\section{THE PERFORMANCE OF OFDM SYSTEM IN AWGN CHANNEL}

This section includes the BER performance of uncoded and coded OFDM over AWGN channel.

The OFDM system parameters used according to IEEE Std 802.16.1a-2013. The modulation technique is QPSK, FFT length equals 1024 and the cyclic prefix (CP) length equals $1 / 8$ of the symbol length [16]. The performance of the system is determined by the relation between bit error rate (BER) and bit energy to noise ratio $\left(\mathrm{E}_{\mathrm{b}} / \mathrm{N}_{\mathrm{o}}\right)$. Assume that, the desired quality of transmission is $\mathrm{BER}=10^{-4}$ and the objective is to achieve this BER with the minimum power as possible.

\subsection{The BER Performance of uncoded OFDM}

The BER performance of the uncoded OFDM system over the AWGN channel can be represented theoretically by theoretical BER for QPSK equation:

$\mathrm{BER}=0.5 * \operatorname{erfc}(\sqrt{\mathrm{Eb} / \mathrm{No}}) \quad[10]$

And also can be represented practically by simulation as shown in Fig 6.

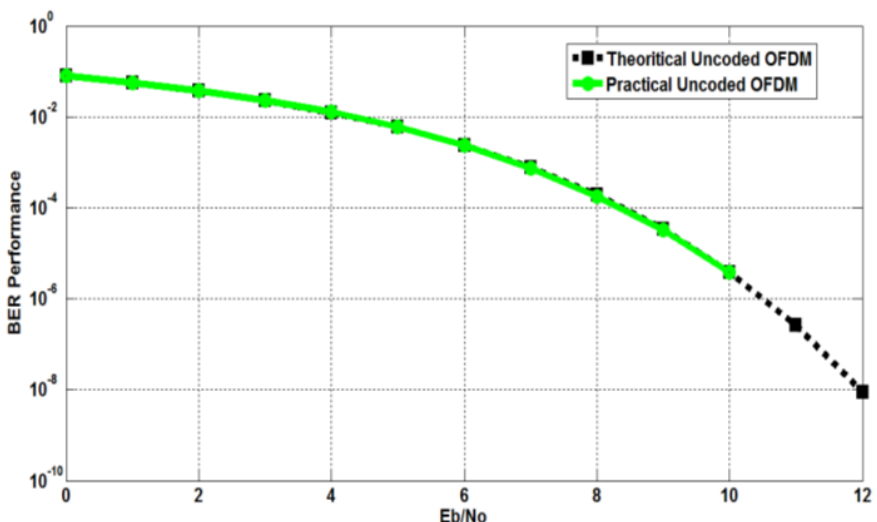

Fig 6: Theoretical and Practical Uncoded OFDM

From Fig.6, the desired BER which is $10^{-4}$ achieved in the uncoded OFDM at $E_{b} / N_{o}=8.2 \mathrm{~dB}$. So the target is to achieve the same quality of transmission but at lower power level.

\subsection{Uncoded OFDM versus coded OFDM}

This section discusses the performance improvement for OFDM system using different channel coding techniques.

\subsubsection{Reed Solomon coded OFDM}

4.2.1.1 The Performance of RS Codes Depends on the Redundancy and the Codeword Length This section shows the performance of Reed Solomon code through AWGN channel model, by changing the codeword length and keeping the code rate with the optimum value which assumed to be 0.7 as mentioned in reference [5].

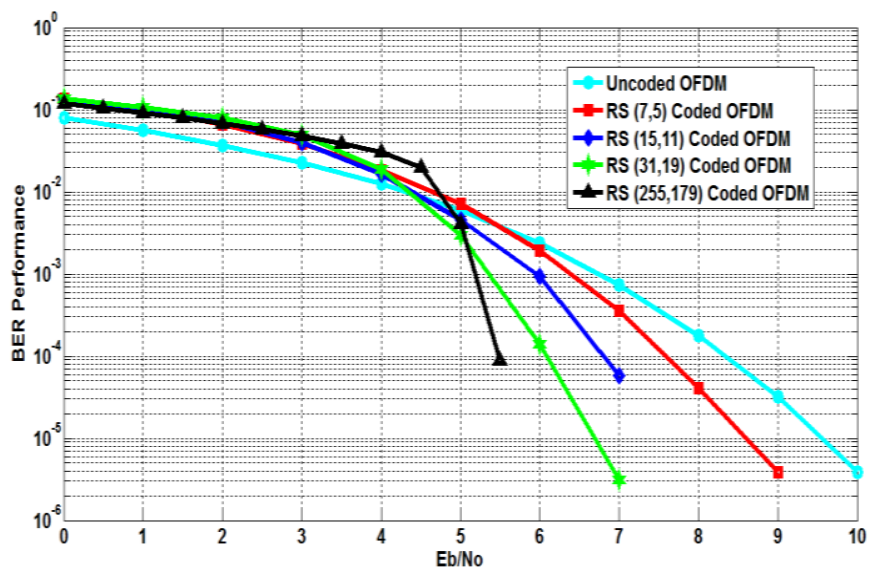

Fig 7: Reed Solomon coded OFDM with Different Lengths

According to Fig 7, the RS coded system performance improved with increasing its codeword length. So the best performance achieved with RS $(255,179)$ which achieve the desired BER at $\mathrm{E}_{\mathrm{b}} / \mathrm{N}_{\mathrm{o}}=5.5 \mathrm{~dB}$, so achieve approximately 2.7 $\mathrm{dB}$ as coding gain. Coding gain refers to the saving in $\mathrm{E}_{\mathrm{b}} / \mathrm{N}_{0}$ due to the used coding technique.

\subsubsection{Shortened RS Coded OFDM}

From Fig 8, the shortened RS code, which have approximately the same rate as the original code, will result approximately the same bit error performance, it provides the desired BER at the same power level that as the original code, besides reducing the complexity and decoding time. 


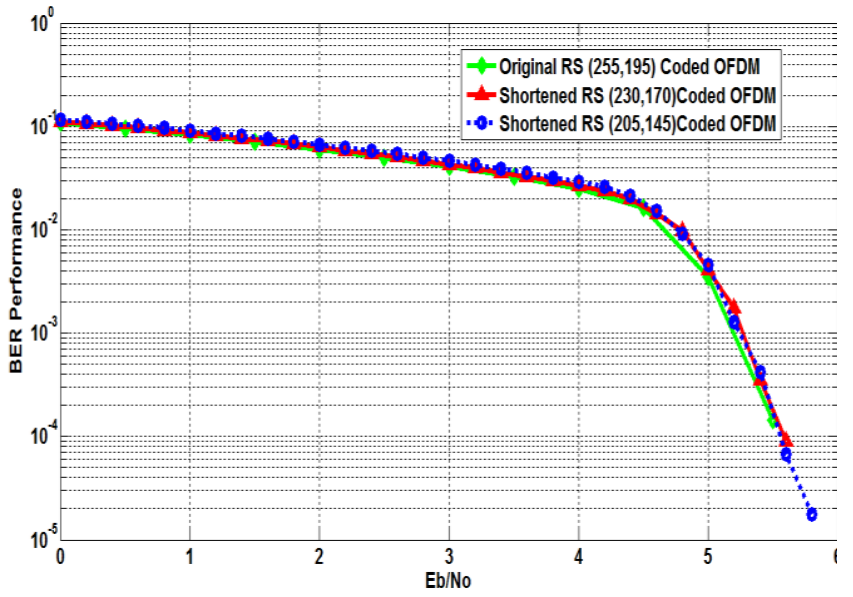

Fig 8: Shortened RS Coded OFDM

\subsubsection{Convolutional coded OFDM}

In the simulation of OFDM using convolutional coding technique, there are two an extra points must be discussed:

\subsubsection{A New Method to Input the Data to the Convolutional Encoder}

In the normal way, the input data enters to the convolutional encoder, then at the decoder, There exist a trace back delay which is a delay that the decoder need to create the complete view about the trellis diagram that used in the decoding process, and after this trace back delay, the decoder begin to output the decoded data. So, due to this delay the decoded data contains number of zero bits at the first, which equal to the trace back length in bits, followed by the decoded data that refers to the original data, but with missing of the last number of bits in the original data. So the decoded data will be the original data with missing of some bits.

For example with convolutional code with $1 / n=1 / 2$ and $K=3$ and generator polynomial $[7,5]$ with a trace back delay $=3$ :

msg_entered_to_encoder $=$

$$
\begin{array}{llllll}
1 & 0 & 1 & 1 & 1 & 0
\end{array}
$$

encoded_msg =

$$
\begin{array}{llllllllllll}
1 & 1 & 1 & 0 & 0 & 0 & 0 & 1 & 1 & 0 & 0 & 1
\end{array}
$$

decoded_msg $=$

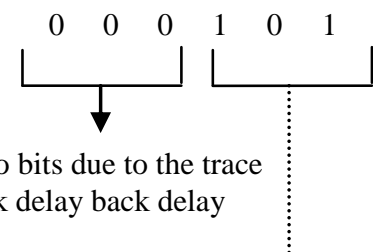

The original data with missing of the last 3 bits

In the new method, the input to the encoder will be the input data followed by number of zero bits equal to the trace back delay length. So that, at the decoder with certain trace back length, after the trace back delay the decoded data will contain firstly number of zero bits equal to delay length, followed by the decoded data that refer to the original data without any missing in the original data but ignoring the last bits in the data which is the previously added zero bits. So that, with this new method, the whole original data will appear in the decoded data without missing. For the same previous example, by adding 3 zero bits to the data that enter to the encoder:

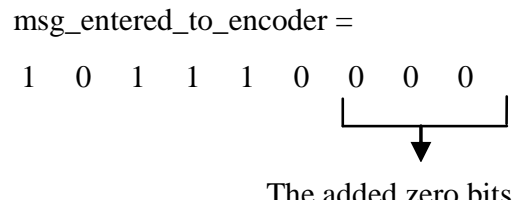

encoded_msg =

$$
\begin{aligned}
& \begin{array}{lllllllllllllll}
1 & 1 & 1 & 0 & 0 & 0 & 0 & 1 & 1 & 0 & 0 & 1 & 1 & 1 & 0
\end{array} \\
& \begin{array}{lll}
0 & 0 & 0
\end{array}
\end{aligned}
$$

decoded_msg =

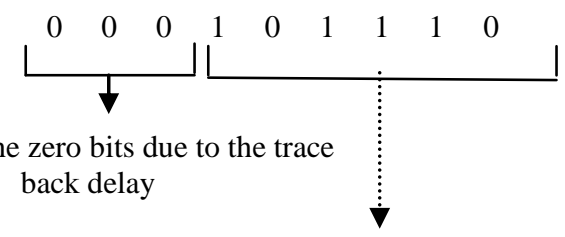

The original data without any missing in the data bits

In the simulation, because of the number of transmitted bits will be very large, the normal way with ignoring the last bits of the data and the new method without ignoring data will provide approximately the same results in the calculation of BER performance. But, the new method will be mainly important in the practical case.

Also it is useful in the concatenated code, where the output of the inner decoder (convolutional) will be entered as input to the outer decoder (Reed Solomon), in the case of using the normal way the output of convolutional decoder, with ignoring the last bits of the data, must be appended with zero bits before entering to the outer decoder (RS), so the decoder also recover the original data with missing of some bits.

But, when using the new method in the concatenated code, the output of the convolutional decoder includes the whole original data, so this output enters directly to RS decoder.

This new method does not affect the system rate, and this is because the very large number of transmitted data with respect to the number of added zero bits. Also it provides a little difference in the BER performance in a certain range of SNR due to the same previous reason as shown in Fig 9. But mainly this method will affect in the practical system.

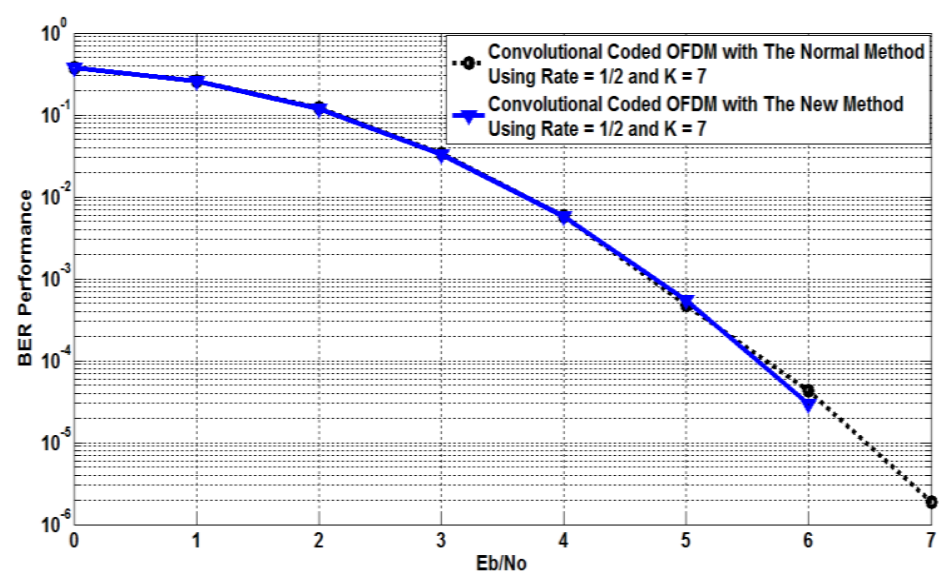

Fig 9: Convolutional Coded OFDM with New Method 


\subsubsection{A new algorithm to Create a Good Convolutional Code}

This algorithm can be used to select the best connection for the convolutional encoder to achieve the large distance that represents a measure to the error correcting capability of the code.

This algorithm start its work by selecting the required rate $1 / \mathrm{n}$ and constraint length $\mathrm{K}$, then testing the all possible connection of the encoder to find the best connection that has a large free distance. From the matlab simulation of this algorithm, to create a good convolutional code, by selecting rate $=1 / \mathrm{n}=1 / 2$ and constraint length $=\mathrm{K}=3$, the I

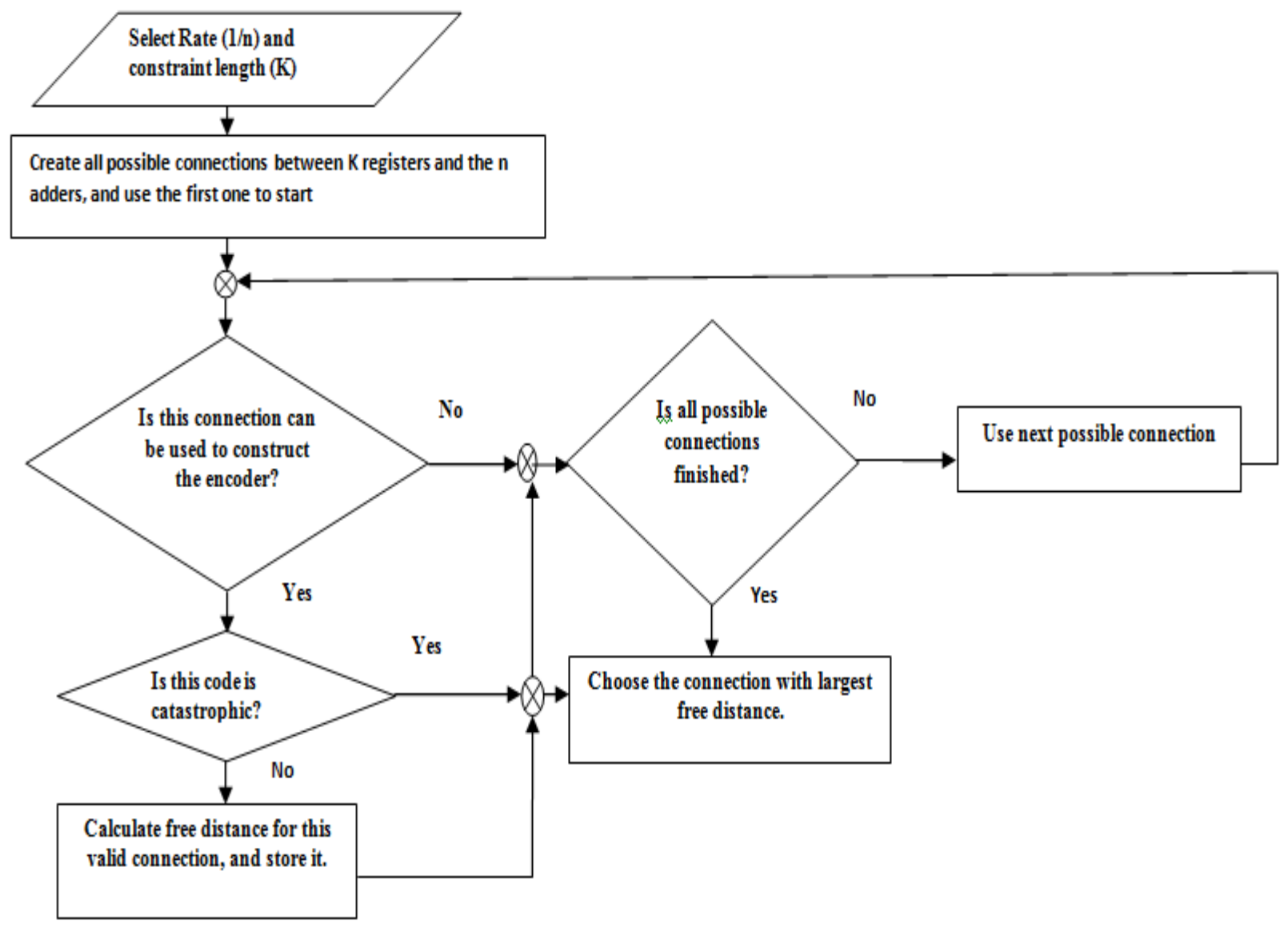

convolutional encoder design with maximum free distance will have the best connection as shows:

good_conv_code =

'n' 'k' 'max dfree' 'best connection vector'

$\begin{array}{lllll}{[2]} & {[3]} & {[5]} & {[5} & 7] \\ {[2]} & {[3]} & {[5]} & {[7} & 5]\end{array}$

So, there are many choices of the encoder circuit connections that have a maximum distance. Fig 10 shows the flowchart that illustrates the simulation process of choosing the good distance convolutional encoder design.

Fig 10: An Algorithm to Select Convolutional Code with Large Distance

\subsubsection{The Performance of Convolutional Codes with Different Parameters}

Here, the convolutional coded system is simulated for different rates and different constraint lengths (K). From Fig 11 , the convolutional code with large constraint length and large rate provides a better performance, but the preferred rate for convolutional code is $1 / 2$ as it will limit some of bandwidth consumption.

The convolutional coded system with rate $1 / 2$ and $\mathrm{K} 7$ will achieve the target BER at $5.5 \mathrm{~dB}$.

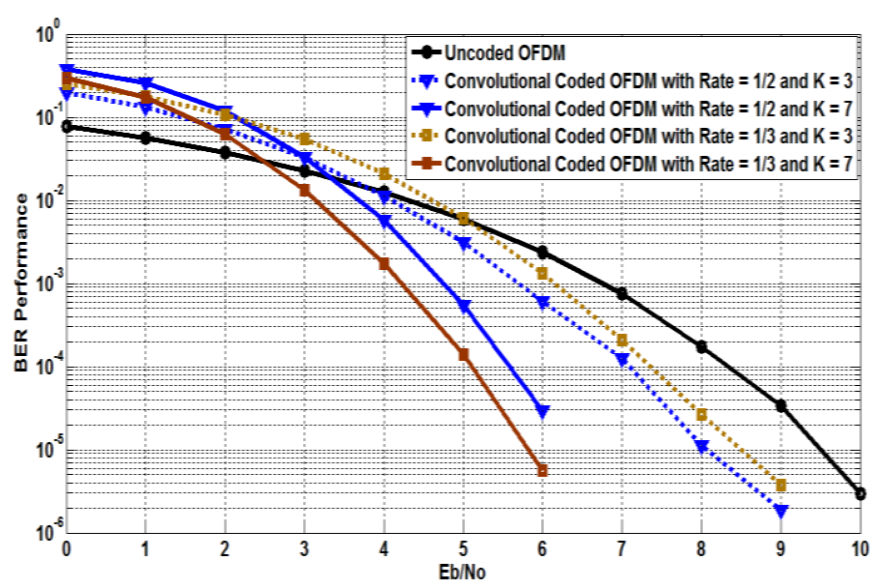

Fig 10: Convolutional coded OFDM 


\subsubsection{Punctured Convolutional Coded System Performance}

Fig 12 examines the performance of the punctured convolutional code with different rates. It appears that, for the punctured convolutional code with rate $2 / 3$, the rate increases but the performance degrades. For the other curves, as the rate approximately remain constant its error correcting capability still fixed and its performance as the case of the original convolutional code, and its benefit is to save some decoding time and complexity by saving or removing only a small number of bits that out from the encoder. So, the punctured convolutional code adjusted to make a balance between its advantage and disadvantage, to have the same performance with less complexity. Finally, this technique can be adjusted according to the application requirements.

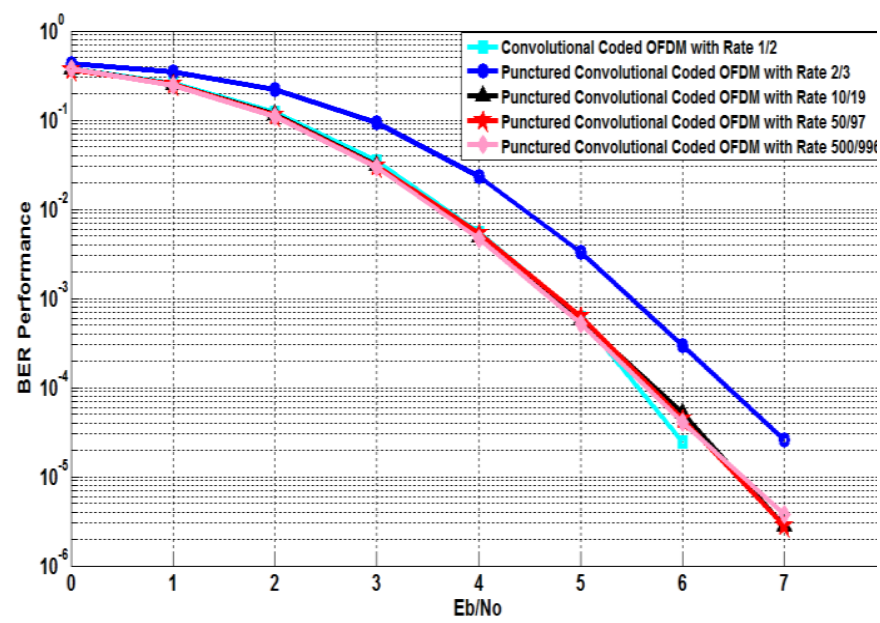

Fig 11: Punctured Convolutional Coded System

\subsubsection{Concatenated coded OFDM}

Firstly, the Reed Solomon code concatenated with the convolutional code. The concatenation is done between two moderate codes the first is RS code, with lower redundancy so more simple, and the second is the convolutional code with rate $1 / 2$, which use Viterbi algorithm that makes it also simple in computation. Fig 13 shows that, the code that uses RS $(255,239)$ and the other that use RS $(255,195)$ provides the desired BER at $3 \mathrm{~dB}$ and $3.1 \mathrm{~dB}$ respectively, with coding gain approximately equal $5.2 \mathrm{~dB}$ than the uncoded case.

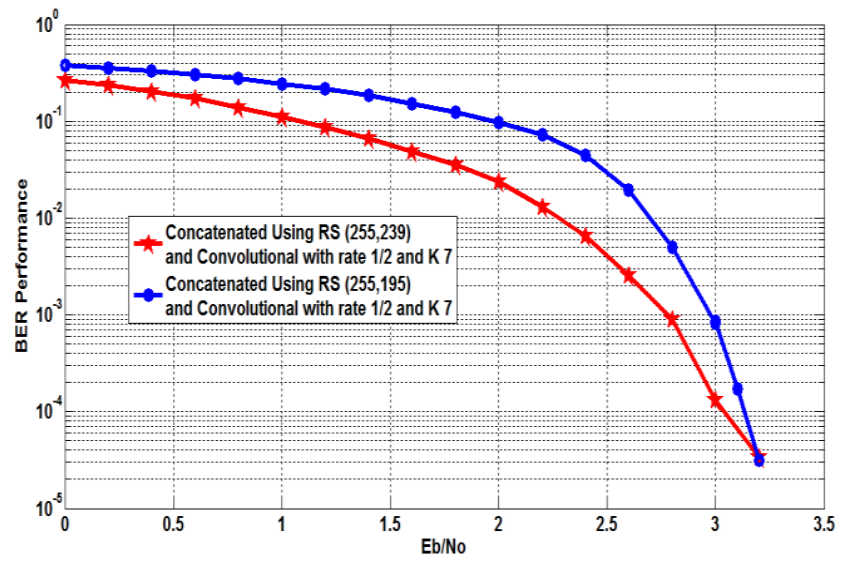

Fig 12: Concatenated Coded OFDM

\subsubsection{Concatenated Coded System with Less Decoding Complexity and Time}

The system performance can be enhanced by using the shortened version of RS $(255,239)$ which is RS $(204,188)$ and the punctured version of convolutional code with rate $1 / 2$ which is punctured convolutional with rate 50/97, as shown in Fig 14.

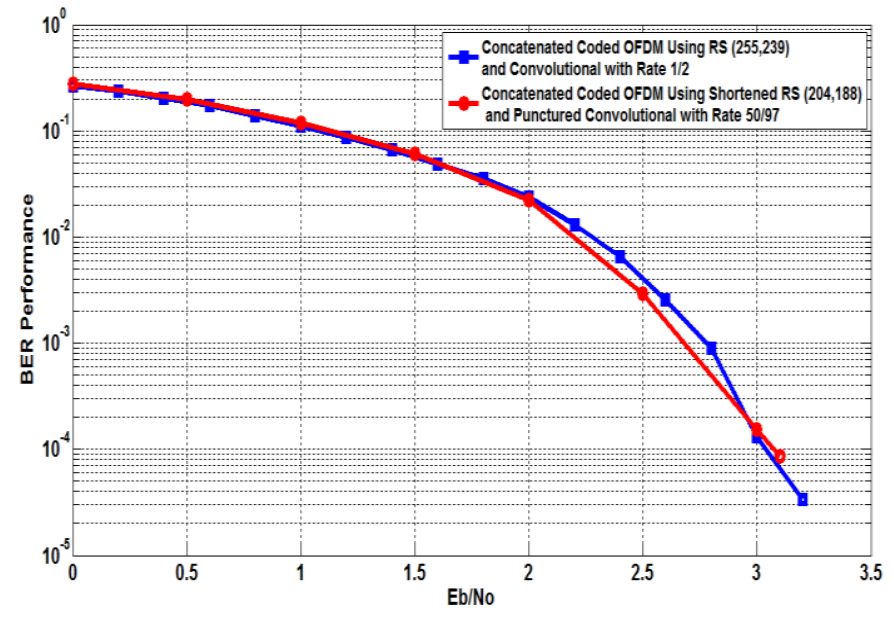

Fig 13: Concatenated Coded OFDM Using the Shortened RS and the Punctured Convolutional Codes

From the previous figure, the modified concatenated coded OFDM system, with shortened RS and punctured convolutional, provides approximately the same performance as that the normal concatenated code but with less complexity and decoding time.

\subsubsection{Interleaved Concatenated coded OFDM}

The use of interleaver in the concatenated system will improve the error performance. Fig 15 shows that the performance for Interleaved Concatenated code OFDM system, the interleaved concatenated coded OFDM system will achieve the desired BER at $2.6 \mathrm{~dB}$. Which means that there is a coding gain $=5.4 \mathrm{~dB}$ with Interleaved Concatenated OFDM system than the uncoded case.

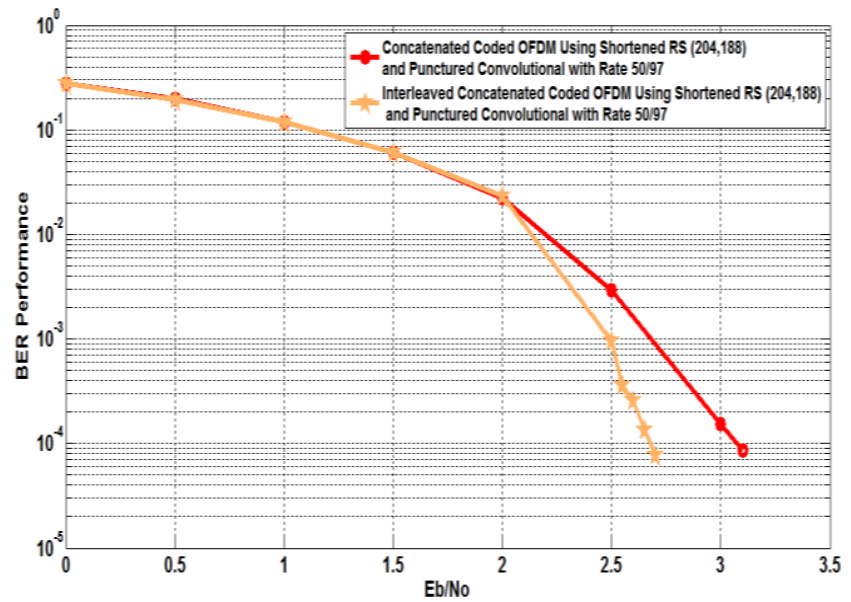

Fig 14: Interleaved Concatenated Coded OFDM

Finally, Fig 16 shows the performance of uncoded versus different types of coded OFDM system, and the used coding technique depends on the available resources and the application requirement. 


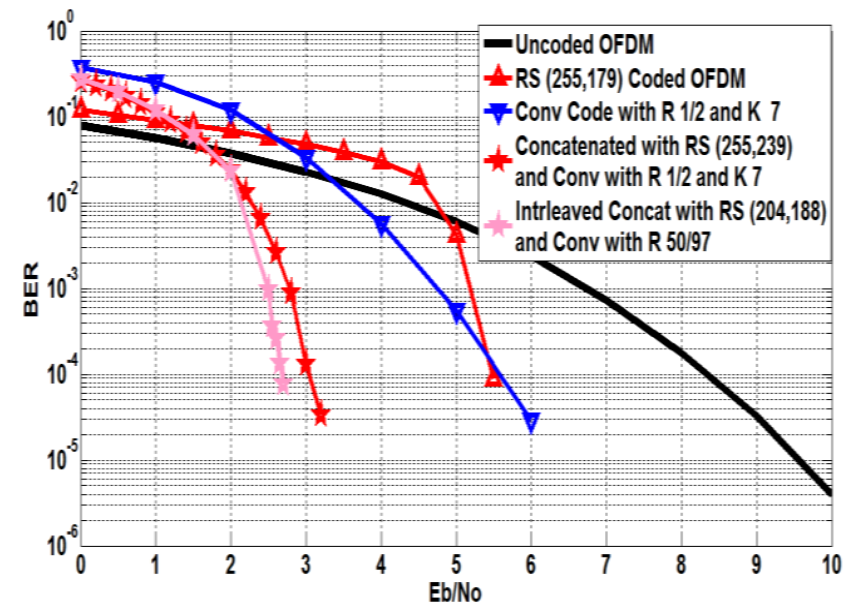

Fig 15: Uncoded OFDM versus coded OFDM

As shown in the previous figures, the different coding techniques improve the performance of OFDM system by reaching lower BER at earlier $\mathrm{E}_{b} / \mathrm{N}_{0}$. But there is a crossover between uncoded and coded performance, which means that , at low values of $\mathrm{E}_{\mathrm{b}} / \mathrm{N}_{0}$, the uncoded system has a lower BER than coded system.

The reason of this crossover is that every code system has some fixed error correcting capability, and if the errors within a block exceed code correcting capability, the code system cannot correct it and perform poorly. Coding techniques, which means adding redundancy, cause high data rate, less energy per bit, and more errors out of the demodulator that can reach and exceed code correcting capability, so cause poor performance. So the benefits of coding in performance appear at reasonable values of $\mathrm{E}_{\mathrm{b}} / \mathrm{N}_{0}$ that compensate the poor performance of the demodulator. Using a powerful coding techniques ,as concatenated codes, cause the crossover occur at earlier point or provide improved performance at lower values of $\mathrm{E}_{\mathrm{b}} / \mathrm{N}_{0}$.[5]

\section{CONCLUSION}

This paper presented the performance of uncoded versus coded OFDM system over AWGN channel. That discusses the effect of different channel coding techniques in the performance of OFDM system. It shows the performance of RS and convolutional codes in OFDM system, then discusses the concatenation between them to provide a better performance at earlier power level. It also discusses the ability of interleaved concatenated codes to provide a more improvement in the system. and its ability to use the shortened and punctured techniques to respond to various systems demands so provide the better performance with the available resources with less decoding complexity and time. It also introduced a new algorithm to choose a good convolutional encoder design with certain rate and memory registers.

As a feature work, the concatenated codes will be applied in the Rayleigh fading channel. And also, his effect on the PAPR will be tested.

\section{REFERENCES}

[1] Husein A.A.Alenzi, Orthogonal Frequency Division Multiplexing, M.Sc Degree in Information Technology at the AHLIA University, 2009.

[2] Sanja Maksimović, Danijela Šikanja,and Željen Trpovski, Single Frequency Networks for DVB-T and Evolution Towards DVB-T2, Scientific Technical Review, 2010 (ok1)

[3] Akhtar Ali Jalbani, SINGLE FREQUENCY NETWORKS, PhD Scholar, Institute for Informatik, University of Goettingen, 2010 (ok4)

[4] Teddy Purnamirza, The Performance of OFDM in Mobile Radio Channel, M.Sc Degree in Engineering at University Technology Malaysia, 2005.

[5] Bernard Sklar, Digital Communications: Fundamentals and Applications, Prentice-Hall Inc., 2001.

[6] Bernard Sklar, Fundamentals of Turbo Codes, 2002.

[7] Jorge Castiñeira Moreira, Patrick Guy Farrell, Essentials of Error Control Coding, Wiley \& Sons Inc., 2006.

[8] Sandeep Katakol, Orthogonal Frequency Division Multiplexing (OFDM), S.D.M College of Engineering and Technology, Vishveshwaraiah Technological University, 2010 (ok2)

[9] Ruhi Khetrapal and Vipul Awasthi, Enhanced BER Performance in Optical OFDM System, International Journal of Modern Engineering and Research Technology,2014 (ok3)

[10] Dushantha N. K. Jayakody, Leonardo O. Iheme, Erhan A. İnce, Coded QPSK-OFDM for Data Transmission over Fading Channels, IEEE, 2010.

[11] Sanjeev Kumar, Ragini Gupta, Bit Error Rate Analysis of Reed Solomon Code for Efficient Communication System, international journal of computer applications, 2011.

[12] Jeff Foerster and John Liebetreu, FEC Performance of Concatenated Reed-Solomon and Convolutional Coding with Interleaving, IEEE 802.16 Broadband Wireless Access Working Group, 2000.

[13] Chih-Peng Li, Wireless Information Transmission System Lab, National Sun Yat-Sen University, 2008.

[14] http://www.allsyllabus.com/aj/note/Information_Science/ Information\%20Theory\%20and\%20Coding/s11/CODIN G\%20FOR\%20BURST\%20ERROR\%20CORRECTION .php

[15] Venkatesan Guruswami, Introduction to Coding Theory: Reed-Solomon, BCH, Reed-Muller, and Concatenated Codes, CMU, 2010.

[16] IEEE Computer Society, and the IEEE Microwave Theory and Techniques Society, IEEE Standard for Wireless MAN-Advanced Air Interface for Broadband Wireless Access Systems, Amendment 2: Higher Reliability Networks, 2013. 